\title{
Interleukin-22 protects against non-typeable Haemophilus influenzae infection: alteration during chronic obstructive pulmonary disease
}

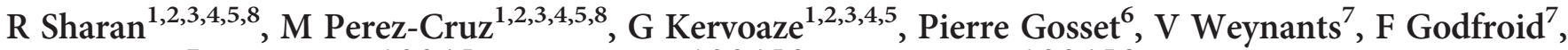

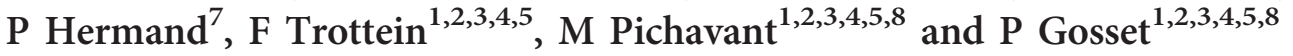

Chronic obstructive pulmonary disease is a major health problem becoming a leading cause of morbidity and mortality worldwide. A large part of these disorders is associated with acute exacerbations resulting from infection by bacteria, such as non-typeable Haemophilus influenzae (NTHi). Our understanding of the pathogenesis of these exacerbations is still elusive. We demonstrate herein that NTHi infection of mice chronically exposed to cigarette smoke (CS), an experimental model of chronic obstructive pulmonary disease (COPD), not only causes acute pulmonary inflammation but also impairs the production of interleukin (IL)-22, a cytokine with potential anti-bacterial activities. We also report that mice lacking IL-22, as well as mice exposed to CS, have a delayed clearance of NTHi bacteria and display enhanced alveolar wall thickening and airway remodeling compared with controls. Supplementation with IL-22 not only boosted bacterial clearance and the production of anti-microbial peptides but also limited lung damages induced by infection both in IL-22 ${ }^{-1-}$ and CS-exposed mice. In vitro exposure to CS extract altered the NTHi-induced IL-22 production by spleen cells. This study shows for the first time that a defect in IL-22 is involved in the acute exacerbation induced by NTHi infection during experimental COPD and opens the way to innovative therapeutic strategies.

\section{INTRODUCTION}

Chronic obstructive pulmonary disease (COPD) is an irreversible condition characterized by a progressive decline in lung function. ${ }^{1}$ Being the fifth leading cause of death worldwide, it is mainly caused by tobacco exposure. ${ }^{2}$ Other potential risk factors include exposure to outdoor pollution, occupational dust, and biomass fuels used for cooking. ${ }^{3}$ Inhalation of cigarette smoke (CS) essentially leads to activation of epithelial cells, macrophages, and other innate immune cells including invariant natural killer $\mathrm{T}$ cells. ${ }^{4,5}$ The chronic inflammatory response progressively leads to airway remodelling, ${ }^{6}$ increased mucus production, ${ }^{7}$ and parenchymal destruction in the lungs, ${ }^{8}$ further culminating in irreversible airflow limitation. Consequently, COPD patients are more susceptible to bacterial or viral airway infections. These respiratory infections are the principal cause of acute exacerbations, which invariably scarred the chronic course of COPD. ${ }^{9}$ Airway colonization with bacteria such as Streptococcus pneumoniae, Moraxella catarrhalis, and Haemophilus influenzae contributes to the pathogenesis and clinical course of the disease. ${ }^{10}$ Non-typeable Haemophilus influenzae $(\mathrm{NTHi})^{11}$ not only colonizes the lungs of COPD patients during clinically stable periods, but acquisition of these bacteria is the most important cause of COPD exacerbations. ${ }^{12} \mathrm{NTHi}$ is a Gram-negative coccobacillus that lacks a polysaccharide capsule. It colonizes the upper respiratory tract of up to $75 \%$ of healthy adults and primarily acts as a mucosal pathogen. NTHi infections induce inflammation with prominent release of cytokines and chemokines including interleukin (IL)-1 $\beta$, IL-6, tumor necrosis factor (TNF)- $\alpha$, and CXCL1. In this setting, activation of Toll-like receptor- 4 and -2 is important to trigger effective innate immune response to NTHi. Though NTHi is a well-recognized

\footnotetext{
${ }^{1}$ Université Lille, U1019 - UMR 8204 - CIIL - Centre d'Infection et d'Immunité de Lille, Lille, France. ${ }^{2}$ CNRS, UMR 8204, Lille, France. ${ }^{3}$ Inserm, U1019, Lille, France. ${ }^{4}$ CHU Lille, Lille, France. ${ }^{5}$ Institut Pasteur de Lille, Lille, France. ${ }^{6}$ Service d'Anatomo-pathologie, Hôpital Saint Vincent de Paul, Lille, France and ${ }^{7}$ GSK Vaccines, Rixensart, Belgium. Correspondence: P Gosset (philippe.gosset@pasteur-lille.fr)

${ }^{8}$ These authors contributed equally to this work.

Received 28 September 2015; accepted 24 March 2016; published online 4 May 2016. doi:10.1038/mi.2016.40
} 
respiratory pathogen in COPD patients, ${ }^{13}$ there is little information on the mechanisms and the susceptibility factors involved in COPD exacerbations. Bacterial infection during COPD (both in patients and experimental models) is characterized by an increased influx of immune cells, including neutrophils, ${ }^{14}$ macrophages, ${ }^{15}$ dendritic cells (DCs) ${ }^{16}$ conventional CD8 ${ }^{+}$T cells, ${ }^{17}$ and NKT lymphocytes, ${ }^{5}$ although the immune response is not able to eliminate the pathogens.

Among factors involved in the control of the bacterial infection, the IL-17 cytokine family is crucial to elicit an effective host response, ${ }^{18}$ although its potential role during NTHi infection is still unknown. IL-17A and the frequently associated cytokine IL-22 are key cytokines for the clearance of various pathogens including Gram-negative bacteria. ${ }^{19}$ Among potential mechanisms, both cytokines induce the recruitment of neutrophils and the production of anti-microbial peptides, such as defensin- $\beta$, REG-3 proteins, or S100A9 by epithelial cells ${ }^{14,20}$ and other host defense genes. In the current study, we hypothesized that an altered production of Th17 cytokines might be responsible for reduced bacterial clearance and COPD exacerbation induced by NTHi. We show that mice chronically exposed to CS have an impaired ability to produce IL-22, but not IL-17, following challenge with NTHi. As showed in vitro, this might be related to a direct effect of CS exposure. Using Il22 $2^{-1-}$ mice, we report an important role for IL-22 in host defense against NTHi. Finally, we show that IL-22 supplementation in COPD and $I l 22^{-1-}$ mice restored bacterial clearance and limited lung damages. Collectively, this study pinpoints the potential interest of targeting IL-22 to treat COPD exacerbation induced by respiratory bacteria.

\section{RESULTS}

\section{COPD mice are more susceptible to NTHi infection}

We have previously established that COPD features, including chronic inflammation, lung function decline, and mild emphysema, were induced in mice exposed to CS for 12 weeks. ${ }^{5}$ Mice chronically exposed to CS were intranasally challenged with NTHi to trigger COPD exacerbation (Figure 1a). At the dose used, NTHi induced a comparable weight loss in CS-exposed and air-exposed mice, with a peak at 3 days post infection (p.i.) (Figure 1b). All mice recovered their initial body weight by day 7 . Compared with control, COPD mice exhibited higher bacterial counts in bronchoalveolar lavage fluids (BALs) and lungs 24 and $48 \mathrm{~h}$ p.i. and then, the bacteria were rapidly cleared (Figure 1c and not shown). The delay in bacterial clearance observed in COPD mice was not associated with a defective expression of anti-microbial peptides such as defensin- $\beta 2-3$, LL-37, Reg- $3 \gamma$, Reg-3 $\beta$, and S100A9 (not shown) as assessed by quantification of transcript. At the protein level, infection with NTHi increased the concentrations of S100A9 but not REG- $3 \gamma$ and defensin- $\beta 2$ and there was no difference between control and COPD mice $(P=\mathrm{NS})$ (Supplementary Figure S1 online).
Histological analysis of lung sections (Figure 1d) revealed that, at $24 \mathrm{~h}$ p.i., there was a moderate cell recruitment both in peribronchial, perivascular spaces, and in the alveoli of control mice. At $48 \mathrm{~h}$, the inflammation was lower in the controls and no airway and alveolar remodeling could be evidenced. In COPD mice, NTHi infection induced a greater inflammation in all lung compartments inducing a severe alveolitis marked by alveolar wall thickening. This was also associated with vasculitis and more mucus secretion within the airways (Figure 1d) at $48 \mathrm{~h}$. This is confirmed by a significant difference between the histological scores ( $5.8 \pm 0.8$ vs. $7.2 \pm 0.8$ in control and COPD mice, respectively, at day 2). Although the inflammation and the alveolitis were resorbed in infected control mice, COPD mice still exhibited a strong thickening of the alveolar wall at day 7 post NTHi challenge, whereas the bacteria were no more detectable (not shown).

Altogether, these data demonstrate that COPD mice are more susceptible to NTHi infection and display increased lung inflammation and remodeling.

\section{NTHi exposure triggers a greater inflammation in COPD mice}

As COPD mice exhibited increased bacterial load and enhanced lung inflammation in response to $\mathrm{NTHi}$, relative to control mice, we next characterized immune cells in the lungs and BALs of each group. At $24 \mathrm{~h}$ p.i., we observed an increased total cell number in the BALs and lungs of infected COPD mice, compared with controls (Figure 2a). The nature and the activation status of inflammatory cells were analyzed by flow cytometry as shown in the Supplementary Figure S2. In contrast with alveolar macrophage, an increased number of DCs $(P<0.05)$ and a trend for a higher neutrophil number in the BALs and lungs was reported in infected COPD mice compared with control mice (Figure 2a, b). This increase was consistent at $48 \mathrm{~h}$ p.i. for neutrophils $(P=0.02)$ (not shown). The number of inflammatory monocytes was also significantly increased at $48 \mathrm{~h}$ p.i. in BALs and lungs of COPD infected mice compared with control mice (data not shown). Infection with NTHi in COPD mice induced a more potent activation of BAL and lung DC, as shown by the greater expression of CD86 and $\mathrm{I}-\mathrm{Ab}$ (Figure 2b).

Moreover, we showed a significantly higher recruitment of both NKT and T cells in the BALs of infected controls and COPD mice $(P=0.001)$ but not in the lungs (Figure $2 \mathrm{c}$ ). Higher levels of CD69 expression (Figure 2d) but not of CD25 (not shown) were observed in NKT cells from the BALs in both control and COPD infected mice compared with not-infected mice, whereas CD69 expression in conventional Tlymphocytes was only increased by NTHi in the lung. Although not significant, there is a trend for an increased level of CD69 in NKT from infected COPD mice compared with controls. In contrast, the recruitment of NK cells tend to be lower in infected COPD mice (not shown).

Overall, increased susceptibility to NTHi infection in COPD mice cannot be explained by a defect in the recruitment and/or the activation of innate and adaptative immune cells. 
CS-exposed mice infected with NTHi shows an impaired expression of IL-22

Inflammatory (IL-1 $\beta$, IL-6, IL-23, and TNF- $\alpha$ ) and immunomodulatory (IL-10, IL-13, IL-17, IL-22, and IFN- $\gamma$ ) cytokines were upregulated after infection with NTHi in the BALs and lung lysates of control mice at 24 and $48 \mathrm{~h}$ p.i.. In COPD mice $24 \mathrm{~h}$ p.i., with the exception of IFN- $\gamma$, significantly higher levels of IL-17 (Figure 3a), IL-1 $\beta$, IL-6, and TNF- $\alpha$ a

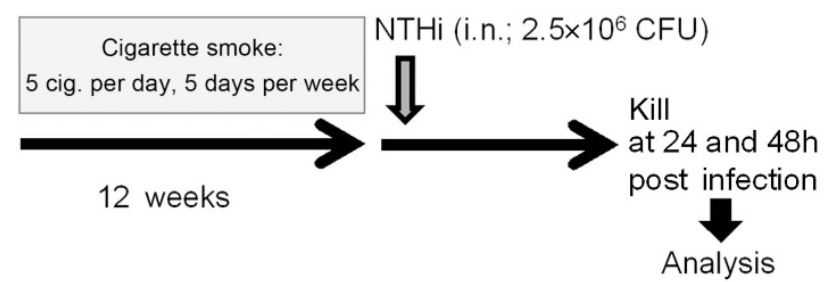

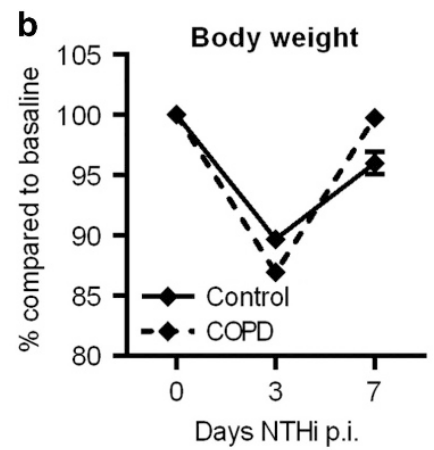

C
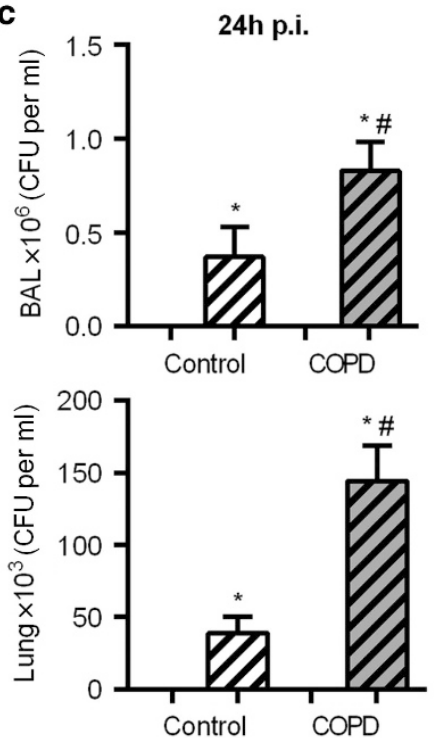

d
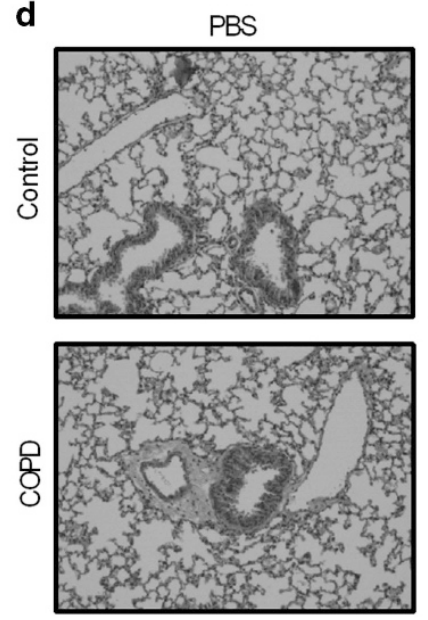
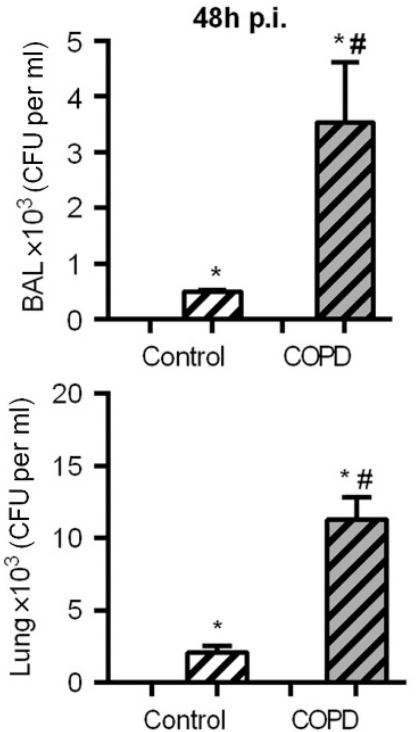

Control

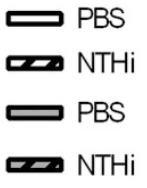

$\Longrightarrow$ NTHi
NTHi 24h p.i.
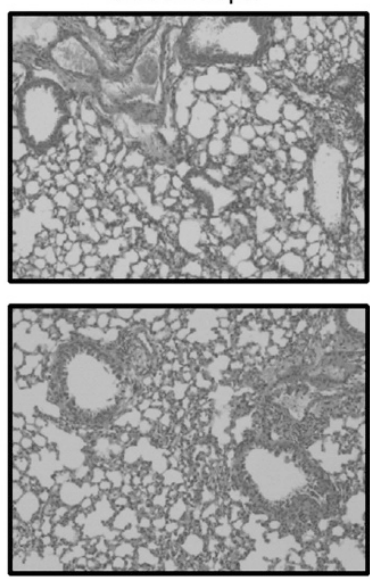
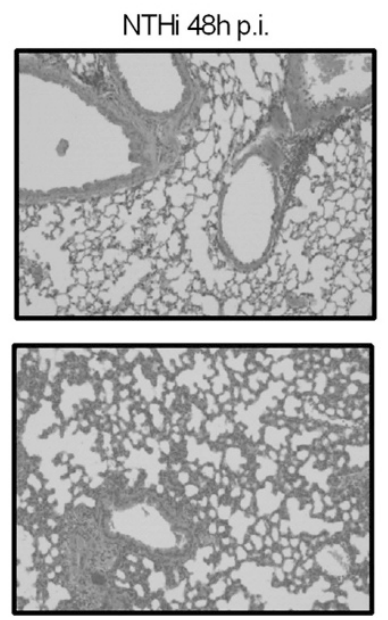

Figure 1 COPD mice are more susceptible to NTHi infection. (a) To assess the impact of smoking on NTHi infection, mice were first exposed to CS for 12 weeks followed by intranasal challenge with NTHi $2.5 \times 10^{6} \mathrm{CFU}$. (b) Body weight loss (expressed as a percentage from body weight at day 0 ) was calculated at days 3 and 7 post NTHi challenge. (c) Mice were killed at 24 and $48 \mathrm{~h}$ post NTHi challenge to assess the CFU counts in BAL and lungs. (d) Lung histopathology of control vs. COPD mice challenged with PBS or with NTHi $2.5 \times 10^{6} \mathrm{CFU}$ at 24 and $48 \mathrm{~h}$ post NTHi challenge. Data are expressed as mean \pm s.e.m. $(n \geq 4)$. ${ }^{*} P<0.05$ compared with PBS; ${ }^{*} P<0.05$ compared with NTHI-infected control mice. BAL, bronchoalveolar lavage fluid; CFU, colony-forming units; COPD, chronic obstructive pulmonary disease; CS, cigarette smoke; NTHi, non-typeable Haemophilus influenzae; PBS, phosphate-buffered saline. 

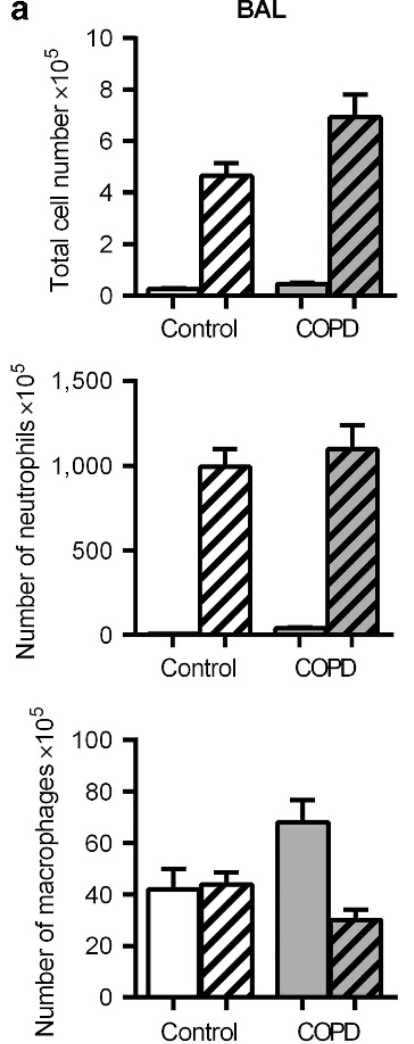

C
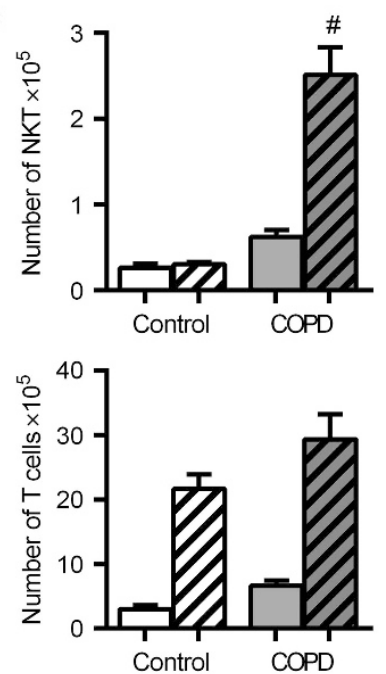

Lung
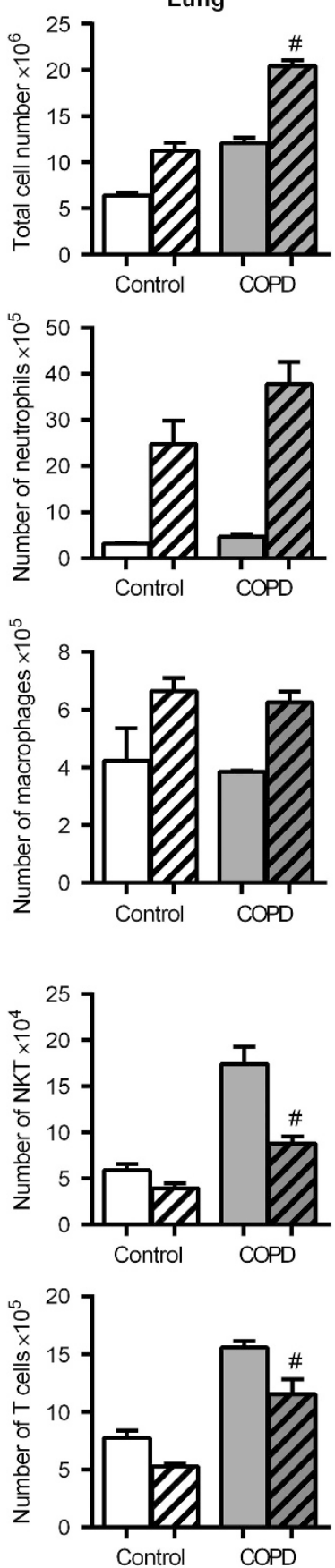
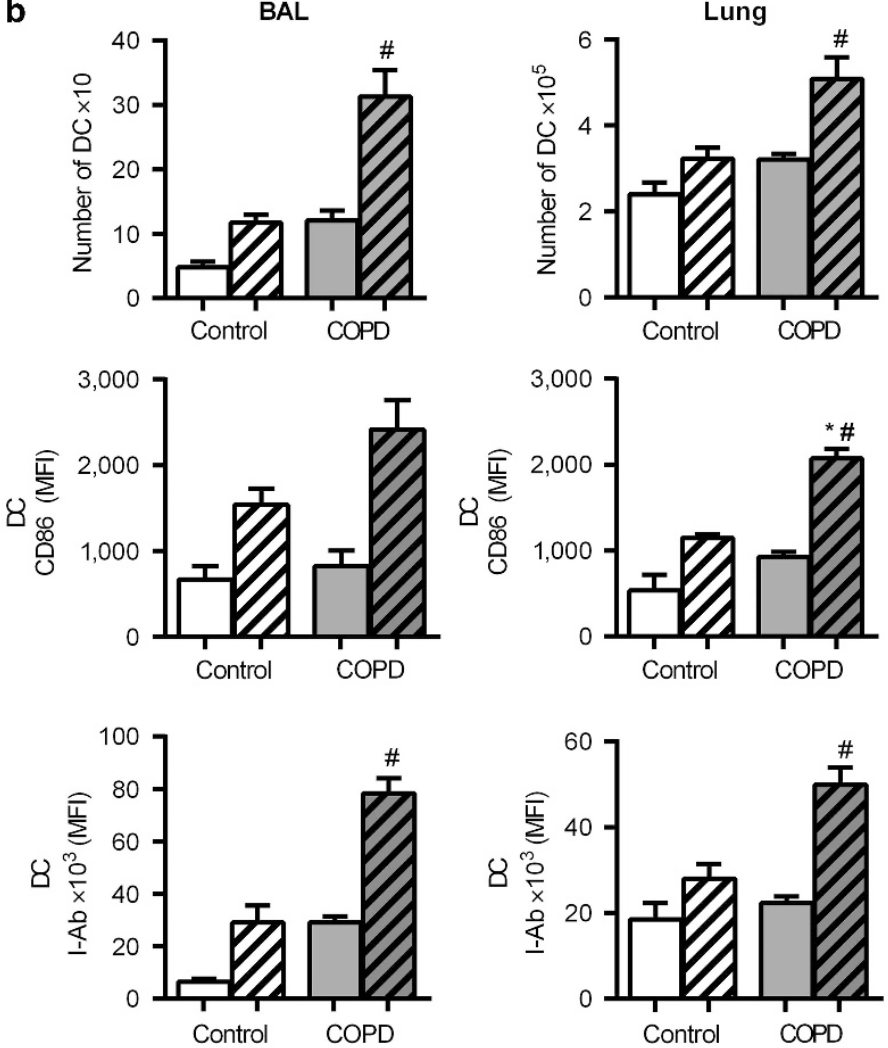

d
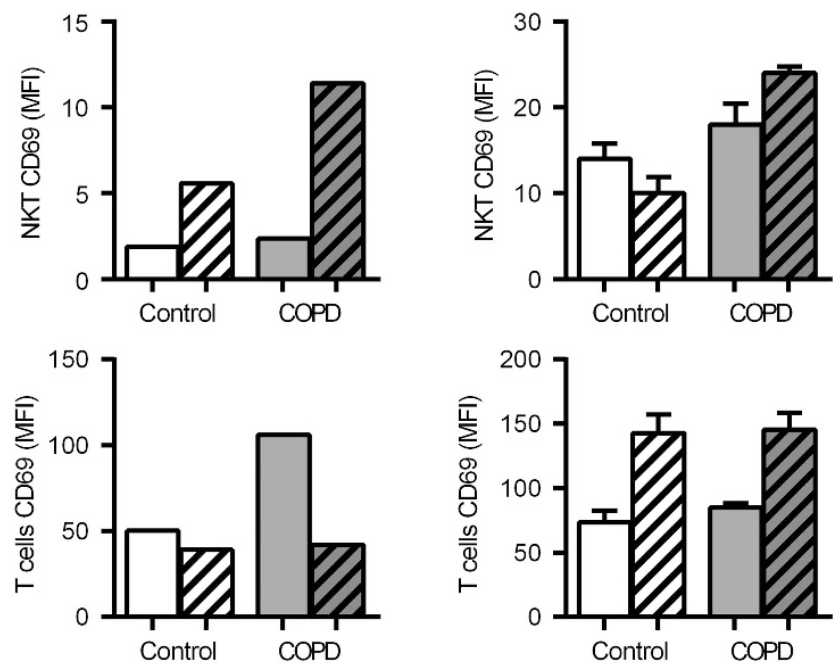

Figure $2 \mathrm{NTHi}$ exposure triggers a greater inflammation in the lung of COPD mice. (a) The total number of recruited cells, absolute number of neutrophils, and macrophages in BALs and lungs of control vs. COPD mice infected or not with NTHi $2.5 \times 10^{6} \mathrm{CFU}$ at $24 \mathrm{~h}$ post challenge. (b) Absolute number of dendritic cells and MFI of the activation markers (CD86 and I-Ab) of dendritic cells. (c) Absolute number of NKT and T cells. (d) MFI of the activation marker CD69 in NKT and T cells from BAL and lungs of COPD infected mice. The data are expressed as mean \pm s.e.m. ( $n \geq 3$ ). ${ }^{*} P<0.05$ compared with PBS; ${ }^{\#} P<0.05$ compared with NTHI-infected control mice. BAL, bronchoalveolar lavage fluid; CFU, colony-forming units; COPD, chronic obstructive pulmonary disease; MFI, mean fluorescence intensity; NTHi, non-typeable Haemophilus influenzae; PBS, phosphate-buffered saline.

(Figure 3b) as well as IL-10 and IL-13 (not shown) were detected in BALs and lung tissue lysates, compared with control mice. A similar trend was observed at $48 \mathrm{~h}$ (Supplementary Figure S3 and not shown). Of interest, although both at 24 and $48 \mathrm{~h}$, the infection with NTHi significantly increased the concentrations of IL-22 in BALs and lung tissue lysates of control mice, these levels were lower in infected COPD animals (Figure 3a).

To analyze the capacity of inflammatory cells to produce immunomodulatory cytokines, lung mononuclear cells were 
a
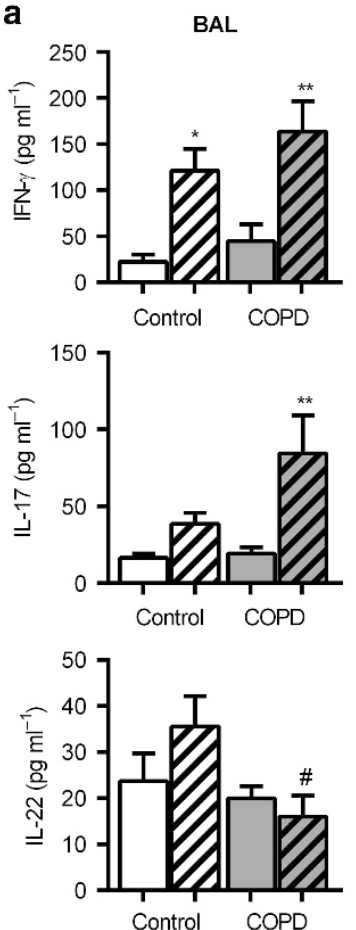

c
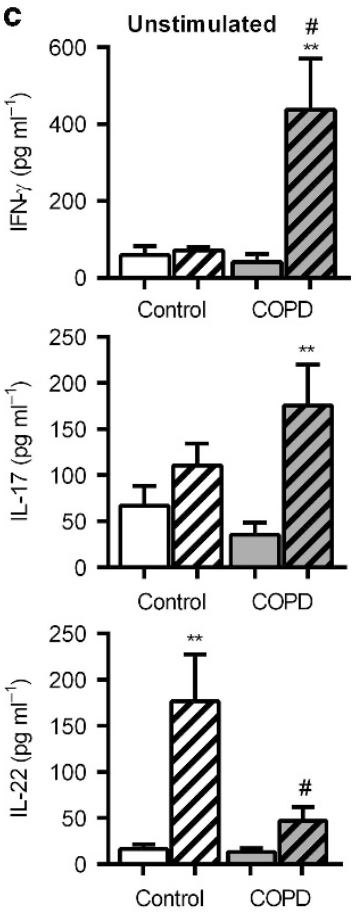
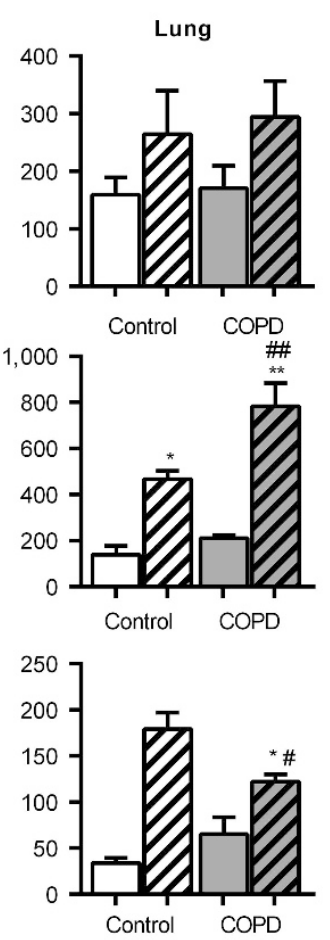

HK NTHi-stimulated
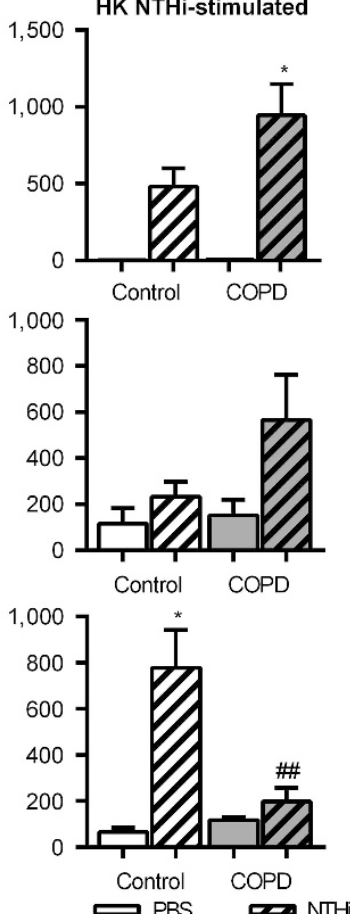

b
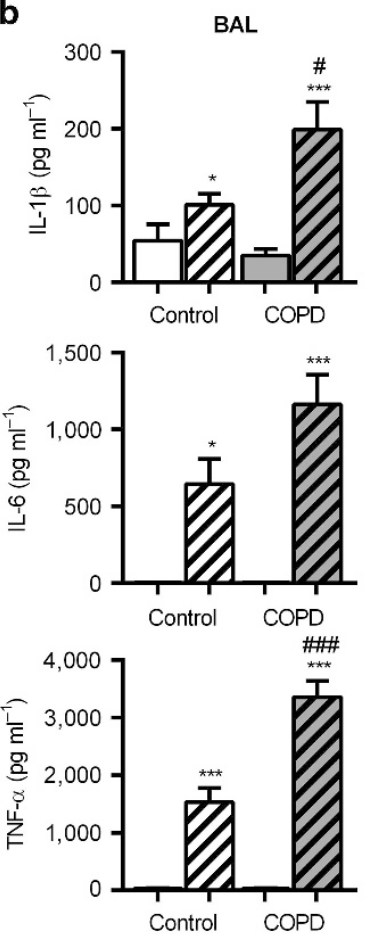

d
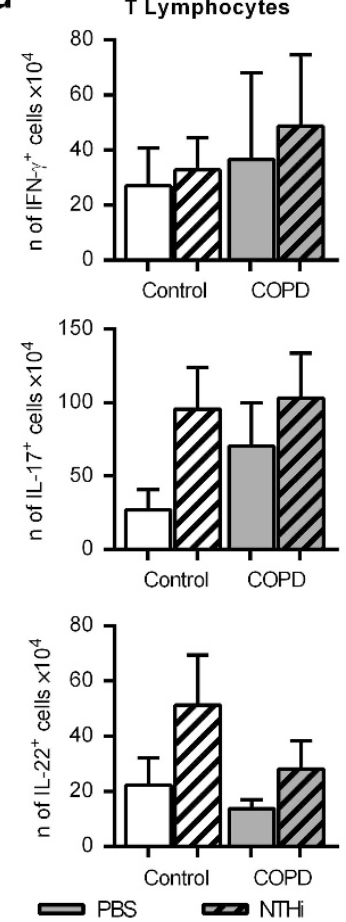
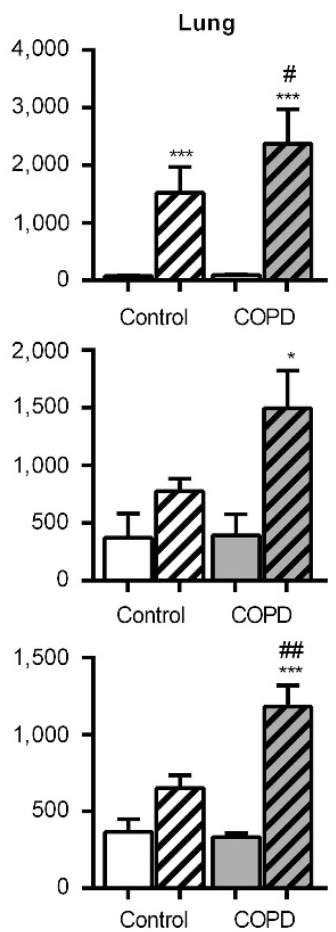

NKT cells
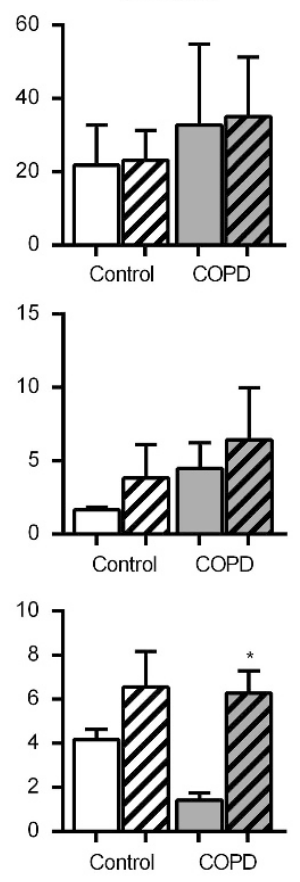

Figure 3 Cytokine profiling in cigarette smoke-exposed mice infected with NTHi. (a, b) The concentrations of the immunomodulatory cytokines (a) IFN- $\gamma$, IL-17, and IL-22 and (b) the inflammatory cytokines IL-1 $\beta$, IL-6, and TNF- $\alpha$ were analyzed using ELISA in BAL and lungs of control vs. COPD mice infected or not with NTHi $2.5 \times 10^{6} \mathrm{CFU}$ at $24 \mathrm{~h}$ post challenge. (c) Lung cells from COPD mice infected with NTHi $2.5 \times 10^{6} \mathrm{CFU}$ per ml were treated with HK NTHi or not (unstimulated). The concentrations of IFN- $\gamma$, IL-17, and IL-22 were analyzed in the supernatants using ELISA. (d) Lung mononuclear cells from COPD mice infected with NTHi $2.5 \times 10^{6} \mathrm{CFU}$ were re-stimulated with PMA/ionomycin for $3 \mathrm{~h}$. Gated T lymphocytes and NKT cells were analyzed for intracellular IFN- $\gamma$, IL-17, and IL-22 production. The data are expressed as mean \pm s.e.m. $(n \geq 3)$. ${ }^{*} P<0.05$, ${ }^{* *} P<0.01$, ${ }^{* \star *} P<0.001$ compared with PBS; ${ }^{*} P<0.05$, \#\# $P<0.01$, \#\#\# $P<0.001$ compared with NTHi-infected control mice. BAL, bronchoalveolar lavage fluid; CFU, colony-forming units; COPD, chronic obstructive pulmonary disease; ELISA, enzyme-linked immunosorbent assay; HK, heat-killed; IFN, interferon; IL, interleukin; MFI, mean fluorescence intensity; NTHi, non-typeable Haemophilus influenzae; PBS, phosphate-buffered saline; PMA, phorbol 12-myristate 13-acetate; TNF, tumor necrosis factor. 


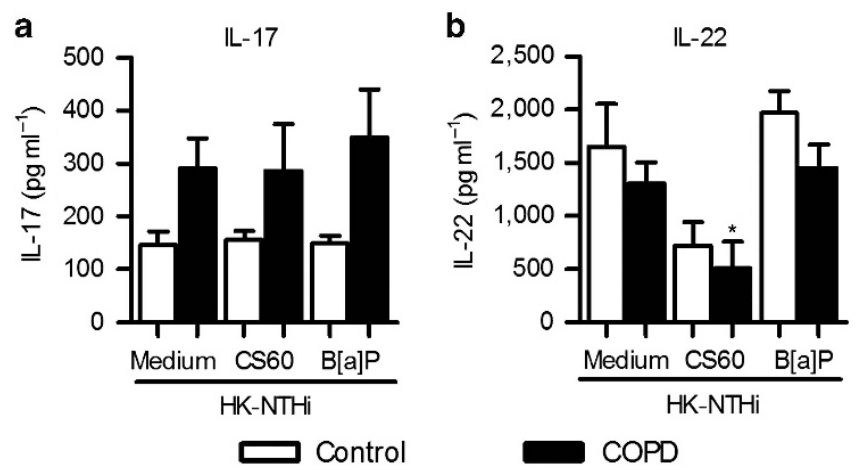

Figure 4 Exposure to CS alters the IL-22 production in COPD by an AhR non-dependant mechanism. To analyze in COPD mice the role of CSE and the implication of AhR in the defect of NTHi-induced IL-22 production, the concentrations of immunomodulatory cytokines; IL-17 (a) and IL-22 (b) were analyzed in the supernatants of spleen cells from control and COPD mice collected $24 \mathrm{~h}$ post challenge with NTHi $\left(2.5 \times 10^{6} \mathrm{CFU}\right)$ at $24 \mathrm{~h}$ post challenge. Mononuclear cells $\left(2 \times 10^{6}\right.$ cells $)$ were stimulated in vitro with HKNTHi with or without CSE or the AhR ligand B[a]P. The data are expressed as mean \pm s.e.m. $(n \geq 4)$. ${ }^{*}: P<0.05$ compared with the medium with cells from COPD mice. AhR, aryl hydrocarbon receptor; BAL, bronchoalveolar lavage fluid; B[a]P, Benzo-a-Pyrene; CFU, colonyforming units; COPD, chronic obstructive pulmonary disease; CS, cigarette smoke; CSE, CS extract; IL, interleukin; NTHi, non-typeable Haemophilus influenzae.

restimulated in vitro with heat-killed (HK)-NTHi. The activation with HK-NTHi significantly augmented the production of IFN- $\gamma$, IL-13, IL-17, and IL-22 in lung cells from infected controls compared with the phosphate-buffered saline mice at $24 \mathrm{~h}$ (Figure 3c) and $48 \mathrm{~h}$ (Supplementary Figure S3 and not shown) p.i. In infected COPD mice, higher levels of IFN- $\gamma$, IL-17 (Figure 3c), IL-13, and TNF- $\alpha$ (not shown) were detected compared with the controls $(P<0.05$ for IFN- $\gamma$ and IL-17). In contrast, the levels of IL-22 were significantly lower in both unstimulated and HK-NTHi stimulated lung cells at 24 and $48 \mathrm{~h}$ as compared with controls (Figure $3 \mathrm{c}$ ).

In order to identify which cells are deficient in the production of IL-22, we analyzed lung cells by intracellular flow cytometry after $3 \mathrm{~h}$ re-stimulation with phorbol 12 -myristate 13 -acetate and ionomycin. The percentage of IFN- $\gamma^{+}$and IL- $17^{+} \mathrm{T}$ and NKT cells did not differ in infected COPD mice compared with controls. In contrast, the number of IL- $22^{+} \mathrm{T}$ cells in COPD mice was lower than in controls, whereas there was no difference in IL-22 ${ }^{+}$NK and NKT cells in the lungs (Figure 3d and Supplementary Figure S4).

In order to define the impact of CS exposure on the IL-22 production, spleen mononuclear cells were restimulated in vitro with HK-NTHi in the presence or not of CS extract (CSE). The activation with HK-NTHi significantly augmented the production of IL-17 and IL-22 in mononuclear cells (Figure 4). Whereas the levels of IL-17 were higher in supernatants from NTHi-activated mononuclear cells of COPD mice compared with control mice, the levels of IL-22 were lower $(P<0.05)$. As expected, exposure to CSE markedly reduced the concentrations of IL-22 (by $70-80 \%, P<0.05$ ) in comparison with controls (Figure $4 \mathbf{b}$ ). IL-17 levels were not modified by exposure to CSE. As CSE contains several ligands of aryl hydrocarbon receptor (AhR), a nuclear factor involved in the control of IL22 gene, we tested in parallel the effect of a canonical ligand of AhR, benzo[a]pyrene $(\mathrm{B}[\mathrm{a}] \mathrm{P})$. Our data showed that addition of $\mathrm{B}[\mathrm{a}] \mathrm{P}$ did not alter IL-22 secretion in spleen cells, in contrast to CSE.

Altogether, these data showed that CS exposure specifically impairs the expression of IL-22.

\section{IL-22 participates in host defense and in the maintenance of the lung structure during NTHi infection}

Our data suggest that IL-22 might be implicated in lung defense against NTHi. To investigate this further, Il22 $2^{-1-}$ mice and wild-type (WT) mice were challenged with NTHi (Figure 5a). As shown in Figure 5b, IL-22-competent animals cleared the bacteria within $24 \mathrm{~h}$, whereas IL-22-deficient animals had a delayed capacity to do so. In parallel, similar S100A9 and REG3 $\gamma$ mRNA and protein levels were observed in the lungs of $I l 22^{-1-}$ mice and control mice, whereas the defensin- $\beta 2$ was only upregulated in IL-22 $2^{-1-}$ mice (Supplementary Figure S5). In addition, the number of neutrophils was not significantly different in the lung of infected $I l 22^{-1-}$ mice, relative to WT counterparts (Figure 5c). Histological analysis showed that the inflammation was almost solved by $48 \mathrm{~h}$ in WT mice, while the infiltrate was still present around the bronchi and alveoli in $I l 22^{-1-}$ mice (Figure 5d). Moreover, alveolar wall thickening was also noticed in infected $I l 22^{-1-}$ mice but not in controls as illustrated by the histological score (6.2 \pm 0.37 and $4 \pm 0.32$, respectively).

To confirm the role of IL-22, we supplemented $I L-22^{-/-}$ mice with recombinant murine (rm) IL-22 before infection (Supplementary Figure S6). Intratracheal injection of rmIL-22 strongly amplified the bacterial clearance in BALs and lungs in $I L-22^{-1-}$ mice (Supplementary Figure S6B). Moreover, this treatment in infected $I L-22^{-1-}$ mice slightly increased the concentrations of REG3 $\gamma$ but not S100A9 and defensin- $\beta 2$ (Supplementary Figure S7). Supplementation with rmIL-22 markedly decreased neutrophil recruitment in lung but not in BALs from infected $I L-22^{-I-}$ mice $24 \mathrm{~h}$ p.i. (Supplementary Figure S6D).

These results suggest that IL-22 has an important role in host defense and in the maintenance of the lung structure during NTHi infection.

\section{Supplementation with IL-22 blocks the development of NTHi-induced exacerbation in COPD mice}

To confirm the role of IL-22 in COPD exacerbation induced by NTHi, we supplemented COPD mice with rmIL-22 before infection (Figure 6a). Intratracheal injection of rmIL-22 strongly amplified the bacterial clearance in BALs and lungs from COPD mice (Figure 6b). Moreover, this treatment increased the expression of S100A9 in the BALs of both groups, whereas REG-3 $\gamma$ was only upregulated in the control mice and defensin- $\beta 2$ was not modulated (Supplementary Figure S8). Treatment with rmIL-22 markedly decreased neutrophil recruitment $(P<0.05)$ in infected COPD mice $24 \mathrm{~h}$ p.i., whereas it did not in control mice (Figure 6c). Histopathological analysis confirmed that infected 
a
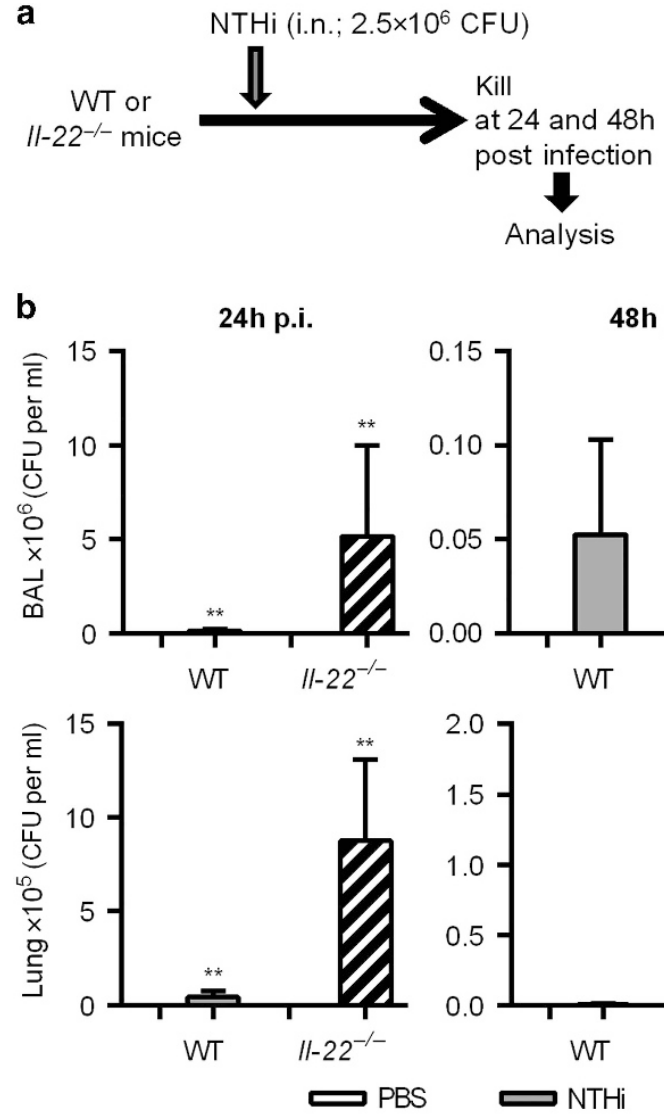

d
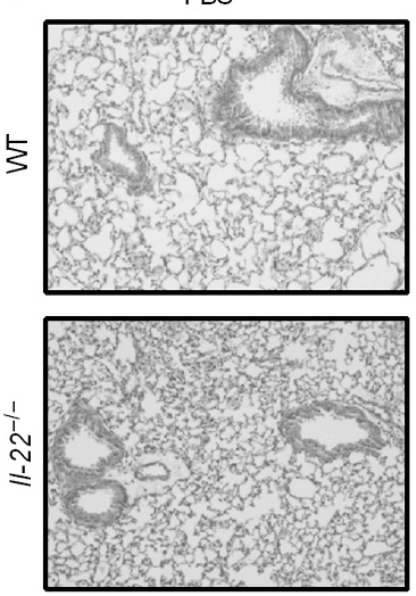

48h p.i.
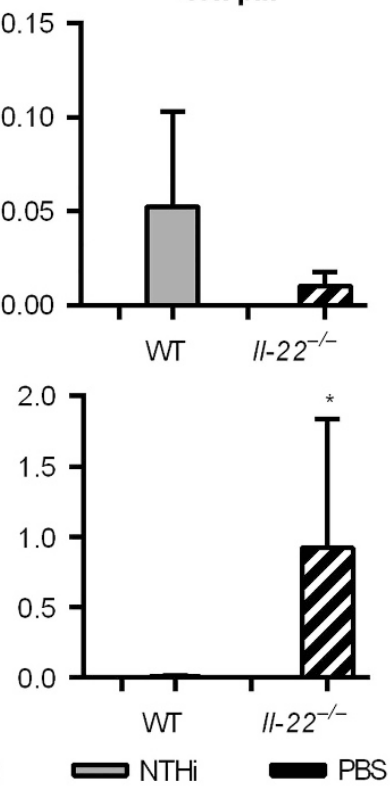

NTHi 24h p.i.
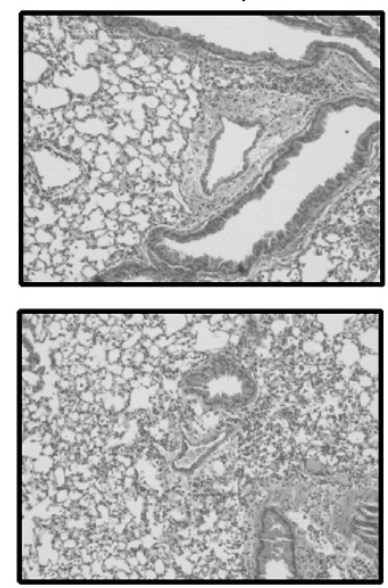

C
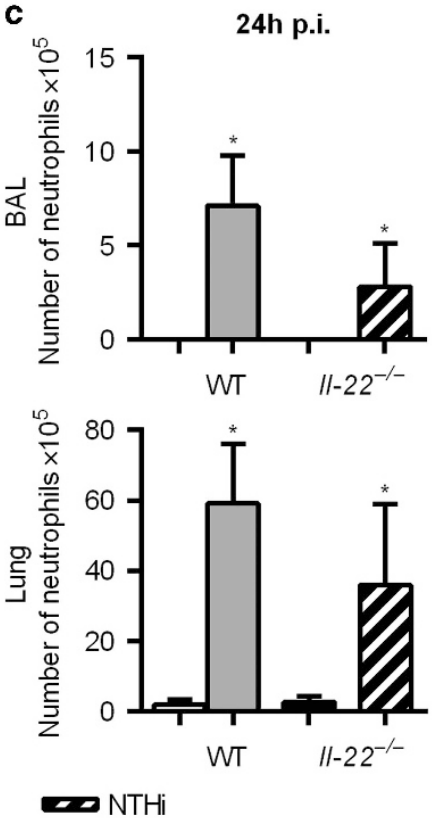

NTHi 48h p.i.
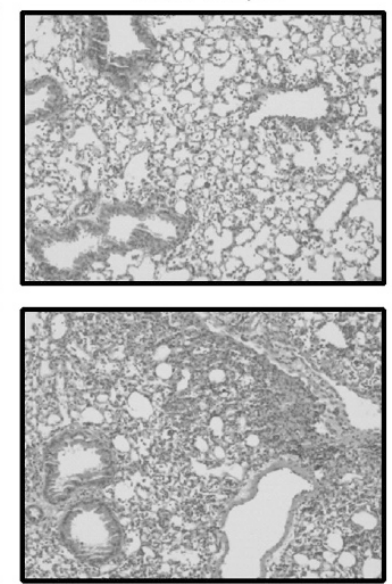

Figure 5 IL-22 participates in NTHi defense. (a) To identify the role of IL-22 in NTHi infection, II-22 ${ }^{-1-}$ and WT mice were challenged with NTHi $2.5 \times 10^{6} \mathrm{CFU}$. (b) Mice were killed at 24 and $48 \mathrm{~h}$ post NTHi challenge to assess the CFU counts in BAL and lungs. (c) The absolute number of neutrophils in BALs and lungs of WT vs. II-22 ${ }^{-1-}$ mice infected or not with NTHi. (d) Upper panel represents histopathology of lung section of WT mice injected with PBS as control and NTHi $2.5 \times 10^{6} \mathrm{CFU}$ at 24 and $48 \mathrm{~h}$ post NTHi challenge. Lower panel represents histopathology of $I I-22^{-1-}$ mice injected with PBS as control and NTHi $2.5 \times 10^{6} \mathrm{CFU}$ at 24 and $48 \mathrm{~h}$ post NTHi challenge. The data are expressed as mean \pm s.e.m. $(n \geq 3) .{ }^{\star} P<0.05$, ${ }^{\star \star} P<0.01 \mathrm{compared}$ with PBS. BAL, bronchoalveolar lavage fluid; CFU, colony-forming units; IL, interleukin; NTHi, non-typeable Haemophilus influenzae; PBS, phosphatebuffered saline; WT, wild type.

COPD mice exhibited higher cell recruitment within the lung at 24 and $48 \mathrm{~h}$ p.i., compared with control mice. As previously shown, infected COPD mice exhibited the features of pneumoniae with alveolitis and a strong vasculitis at $48 \mathrm{~h}$ (Figure 6d). Treatment with rmIL-22 prior to infection markedly reduced the inflammatory infiltrate as well as the lesions targeting blood vessels and alveolar walls in COPD mice as confirmed by the histopathologic score $(7 \pm 0.41$ vs. $4.33 \pm 0.31$ in phosphate-buffered saline- and rmIL-22treated COPD mice, respectively, $P<0.05)$.

To measure the therapeutic effect of IL-22, we supplemented COPD mice with rmIL-22 6 and $18 \mathrm{~h}$ p.i. Intratracheal injection 
a

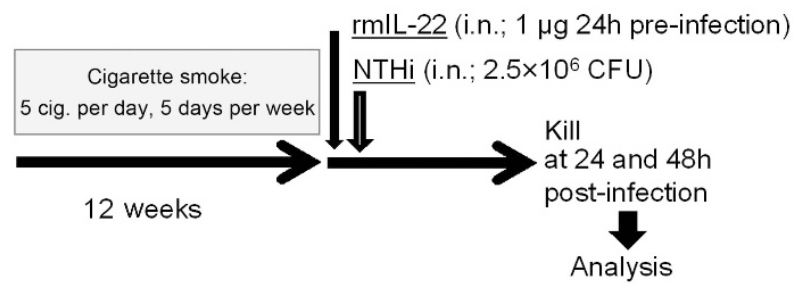

b
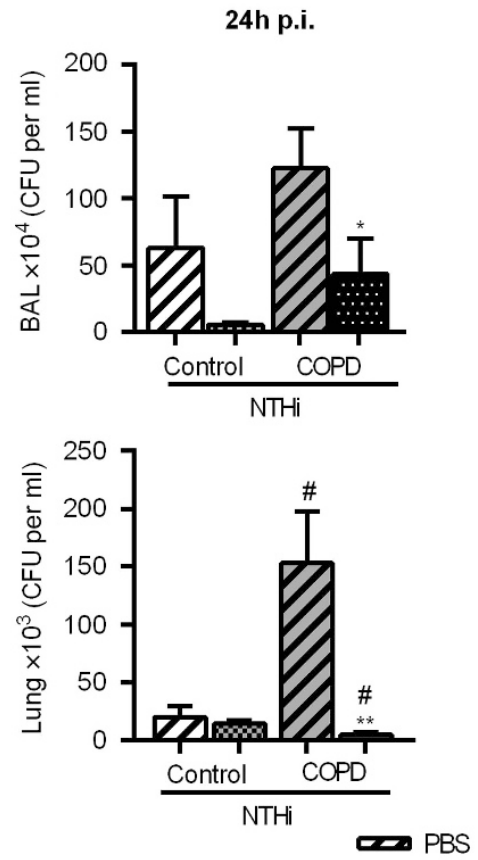

48h p.i.
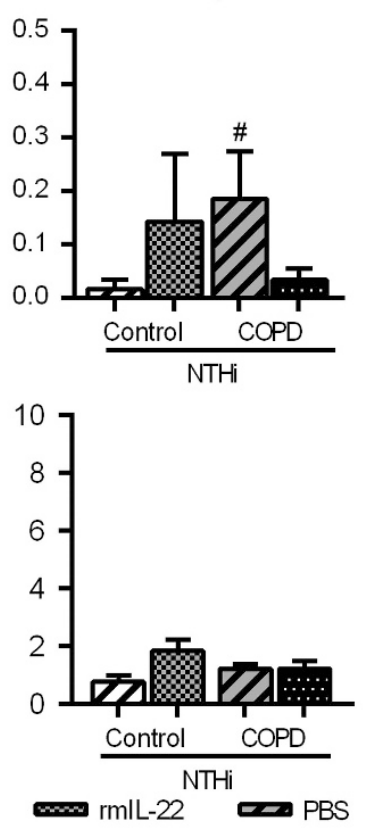

C
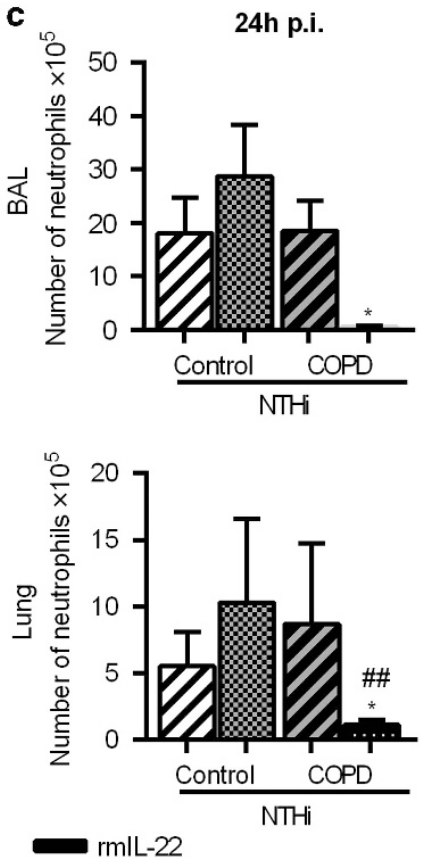

d

$\mathrm{NTHi}$

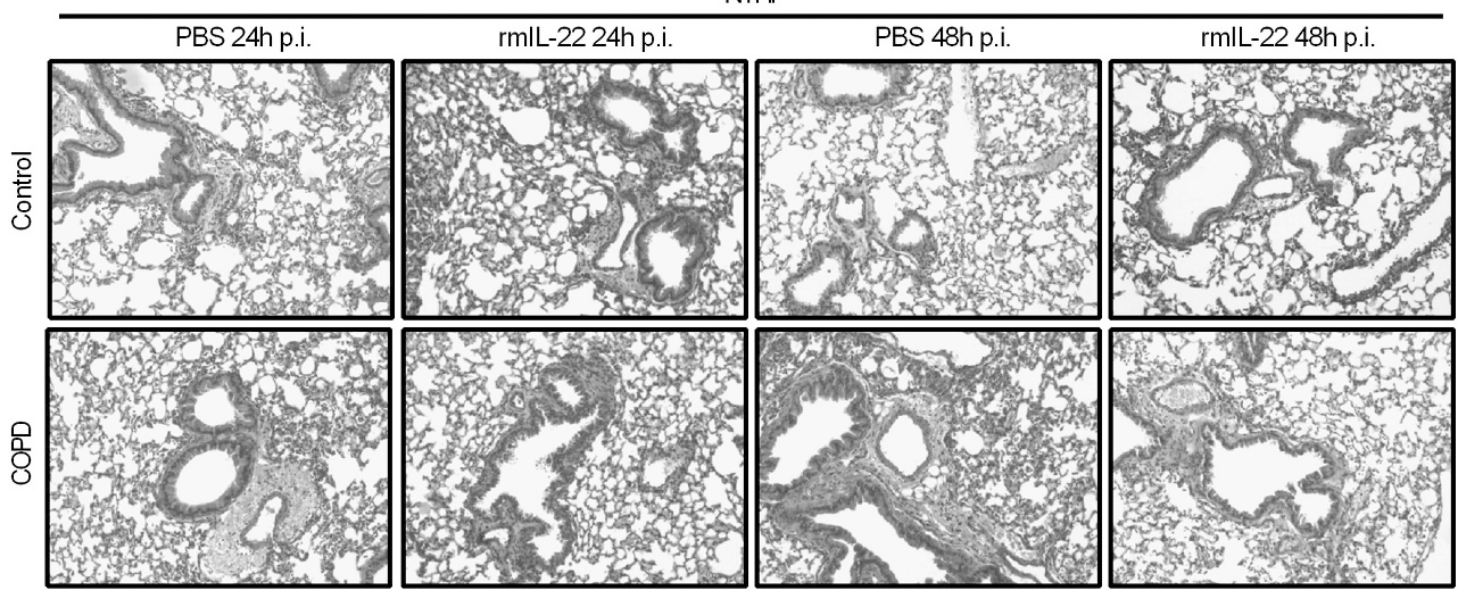

Figure 6 Supplementation with IL-22 limits NTHi infection in COPD mice. (a) To analyze the consequences of supplementing COPD mice with IL-22 on NTHi infection, mice were first exposed, or not, to CS for 12 weeks before being administered intranasally with $1 \mu \mathrm{g}$ of rm IL-22, $24 \mathrm{~h}$ pre-infection. Mice were then intranasally challenged with NTHi $2.5 \times 10^{6} \mathrm{CFU}$. (b) Mice were killed at 24 and $48 \mathrm{~h}$ post NTHi challenge to assess the CFU counts in BAL and lungs. (c) The absolute number of neutrophils in BALs and lungs of control vs. COPD mice infected with NTHi and supplemented or not with rmIL-22. (d) Upper panel represents histopathology of lung section of control mice injected with PBS and rmIL-22 at 24 and $48 \mathrm{~h}$ post NTHi challenge. Lower panel represents histopathology of COPD mice injected with PBS and rmIL-22 at 24 and $48 \mathrm{~h}$ post NTHi challenge. The data are expressed as mean $\pm \mathrm{s}$.e.m. $(n \geq 6)$. ${ }^{*} P<0.05,{ }^{\star \star} P<0.01$ compared with PBS; ${ }^{*} P<0.05$, ${ }^{\# \#} P<0.01$ compared to NTHi-infected control mice. BAL, bronchoalveolar lavage fluid; CFU, colony-forming units; COPD, chronic obstructive pulmonary disease; CS, cigarette smoke; IL, interleukin; NTHi, non-typeable Haemophilus influenzae; PBS, phosphate-buffered saline; rm, recombinant murine. 
of rmIL-22 did not significantly amplify the bacterial clearance in BALs and lungs from COPD mice (data not shown). Treatment with rmIL-22 did not affect the neutrophil recruitment in infected COPD mice $24 \mathrm{~h}$ p.i. (data not shown).

Preventive supplementation with rmIL-22 amplified the clearance of NTHi in COPD mice and limited lung damages resulting from infection.

\section{DISCUSSION}

There is evidence suggesting that COPD is projected to become one of the leading causes of death worldwide. The progression of COPD is worsened by lower airway infection mostly due to respiratory infection by NTHi. Here, we demonstrated that the cytokine response to NTHi was amplified in COPD mice with the marked exception for IL-22. Moreover, we showed that IL-22 is an essential factor in the control of infection by NTHi both in control and COPD mice.

To establish an acute model of exacerbation, mice chronically exposed to CS and presenting the characteristics of $\mathrm{COPD}^{5}$ were challenged with NTHi. We observed an increased bacterial load in the lungs of COPD mice compared with control animals. These higher bacterial counts were associated with marked lung damage and increased mucus secretion. ${ }^{21}$ Our data confirmed that NTHi significantly exacerbated the CS-induced damage. Though the inflammation and the alveolitis was resorbed in air-infected mice, the alveolitis and the pneumoniae was still prominent in infected COPD mice with a strong thickening of the alveolar wall persisting during at least 7 days.

This observation was corroborated by higher influx of inflammatory cells including neutrophils and DC in COPD mice compared with control mice. COPD is often described as a neutrophilic inflammation. Neutrophils through the release of several proteases are involved in lung damages. ${ }^{22}$ The increased presence of neutrophils in lung from COPD mice was probably associated with increased levels of IL- $1 \beta$, IL- 6 , TNF- $\alpha$, and IL-17 compared with control mice. ${ }^{23}$ These cytokines participate in recruitment and activation of inflammatory cells in COPD lung. While IL-1 $\beta$ and TNF- $\alpha$ causes a release of inflammatory chemokines and metalloproteases from macrophages and epithelial cells in COPD lungs, ${ }^{24}$ IL-6 is implicated in neutrophil chemotaxis. ${ }^{23,25}$ IL-17A also induces the production of neutrophil chemoattractants CXCL1 and CXCL8. ${ }^{25,26}$ The implication of IL-17A in the recruitment of neutrophils has been recently reported during NTHiinduced COPD exacerbation. ${ }^{27}$ Altogether, neutrophils promote the lung inflammation and are probably implicated in the airway remodeling observed in infected COPD mice. As the inflammation exists before the infection and persists after the clearance of the bacteria in COPD mice, this recruitment results from both the COPD status and the infection and is not strictly related to the bacterial load.

Though the role of DC during COPD is not clearly understood, previous studies have shown an increased DC infiltration in small airways of COPD patients related to disease severity. ${ }^{28}$ In our model of exacerbation, we observed a significantly higher DC number at $24 \mathrm{~h}$ post NTHi infection in smoke-exposed mice compared with air-exposed mice. These recruited DCs were activated at the site of inflammation as shown by the increased expression of CD86 and $\mathrm{I}-\mathrm{Ab}^{5}$ as well as in alveolar macrophages ${ }^{29}$ and probably, participate at the inflammatory process in COPD. Together, these inflammatory cells seem to cause an irreversible breakdown of lung tissue characteristic of COPD probably through the activation of lymphocytes. ${ }^{5}$ The activation of APC associated with the increased production of IL- $1 \beta$ and IL- 6 in the lung, probably explains the presence of a significantly high number of $\mathrm{T}$ lymphocytes and the increased production of cytokines including IFN- $\gamma$ and IL-17 in the lung of COPD mice. Interestingly, neutralization of IL-17 during NTHi-induced COPD exacerbation reduced the neutrophil mobilization within the lung but did not modulate the bacterial clearance. ${ }^{26}$ This suggests that controlling the inflammatory reaction associated with bacteria-induced exacerbation did not allow to restore an efficient IL-22 production and the elimination of the bacteria.

Interestingly, we detected a marked defect in IL-22 production in COPD mice challenged with NTHi. IL-22 has a crucial role in the defense against lung infections with Gram-negative bacteria. ${ }^{20}$ We have recently observed an altered IL-22 response to S. pneumoniae during COPD both in murine model and in human patients. ${ }^{30}$ With NTHi, we observed an altered production of IL-22 in conventional T lymphocytes but not in NK and NKT cells from infected COPD mice compared with control mice. As mentioned above, this defect is not related with an impaired activation of DC function nor to a defect in the production of pro-Th17 cytokines but is probably linked to an intrinsic defect in the development of Th22 cells. Interestingly, direct exposure to CSE alters the IL-22 production induced by HK-NTHi but not IL-17. We also tested the effect of B[a]P, a component of CS and a well-known ligand of AhR, a nuclear factor involved in IL-22 response. ${ }^{31}$ Treatment with the AhR ligand did not decrease the production of IL-22 even in cells from COPD mice. These data suggest that exposure to CS directly inhibits the NTHi-induced production of IL-22 (but not IL-17) in an AhR-independent manner.

Regarding its role in anti-bacterial defense, IL-22 regulates the antimicrobial activity of lung epithelium, production of chemokines, and survival of Th17 cells. ${ }^{32,33}$ In our study, the level of anti-microbial peptides was not different between control and COPD mice. However, if we relate these levels to the bacterial load, we can suppose that these concentrations are not sufficient in order to control the infection in CS and Il-22 $2^{-1-}$ mice. Moreover, supplementation with rmIL-22 increases the expression of anti-microbial peptides including S100A9 and REG-3 $\gamma$ and at the same time, accelerates the clearance of NTHi. Interestingly, the defective production of IL-22 was associated with an increased dissemination of bacteria in both COPD and $\mathrm{Il}-22^{-1-}$ mice suggesting that IL-22 may also act through the modulation of the epithelial barrier function as previously reported during viral infection. ${ }^{34}$ 
Il-22 $2^{-1-}$ mice challenged with NTHi also presented a weak alteration of neutrophil recruitment associated with a marked effect on bacterial load. Surprisingly, a similar profile was reported in COPD mice treated by rmIL-22. Both models differed by the higher cytokine burst in COPD mice. Treatment with rmIL-22 in COPD mice limits the development of the lung inflammatory reaction (including neutrophil influx) and the remodeling of the airways, a process probably related to the accelerated bacterial clearance. It remains to determine whether the action of IL-22 is linked to the promotion of anti-bacterial peptides, the priming of effector cell (including neutrophil), and/or the maintenance of the barrier function. As we are able to only demonstrate a preventive effect of IL-22 supplementation in our acute model, additional experiments using a more chronic model of infection are required to define the potential curative application of this treatment.

Altogether, we show that defective IL-22 production is involved in COPD exacerbation triggered by NTHi infection. This study identifies IL-22 as a therapeutic target to limit the consequences of NTHi infection in COPD. Restoration of an efficient bacterial clearance and a limitation of the inflammatory reaction could be a step forward for the treatment of COPD exacerbation.

\section{METHODS}

Animals. Male C57BL/6 (WT) or Il-22-1- mice of both sexes, 6-8 weeks old were purchased from Janvier Labs (Le Genest-St-Isle, France) or Jean-Christophe Renauld lab (Brussels, Belgium), respectively. WT mice were daily exposed to CS during 12 weeks. ${ }^{5}$ The sham group was exposed to ambient air. Research cigarettes 3R4F were obtained from the University of Kentucky Tobacco and Health Research Institute (Lexington, KY). After 12 weeks of CS or air exposure, mice were either treated with phosphate-buffered saline or NTHi $\left(2.5 \times 10^{6} \mathrm{CFU}\right.$; i.n.) $(n=4$ per group). All procedures were performed according to the Pasteur Institute, Lille, Animal Care and Use Committee guidelines (agreement number $\mathrm{N}^{0} \mathrm{AF} 16 / 20090$ ) and were approved by the local ethic committee. Experiments were performed at least in triplicate.

Preparation of bacteria and mice infection. NTHi strain 3224A was grown to log-phase in brain-heart infusion broth (AES Laboratoire, Marcy l'Etoile, France) supplemented with haematin and nicotinamide adenine dinucleotide (Sigma, St Louis, MO). The bacterial dose was checked by plating serial dilutions of broth cultures onto chocolate agar plates. For preparation of HK-NTHi), bacteria were grown to a log-phase $\left(\mathrm{OD}_{600 \mathrm{~nm}}=0.7-0.8\right.$ units $)$ and incubated at $56^{\circ} \mathrm{C}$ for $1 \mathrm{~h}$ in a water bath. Broth cultures were then plated onto chocolate agar plates and incubated overnight to check bacterial inactivation.

Mice were anesthetized and then intranasally challenged with $2.5 \times 10^{6}$ colony-forming units (CFU) of NTHi. In IL-22 supplementation studies, $5 \mu \mathrm{g}$ of rmIL-22 was administrated intranasally $24 \mathrm{~h}$ before and $6 \mathrm{~h}$ and $18 \mathrm{~h}$ after NTHi challenge to demonstrate the prophylactic and therapeutic effect of IL-22.

Sample collection and processing. Mice were killed at 24 and $48 \mathrm{~h}$ p.i. BAL fluids, lungs, spleen, and blood were collected and kept on ice till the processing or immediately frozen in liquid nitrogen.

BAL samples were used for cytokine analysis, flow cytometry analysis, and numbering of CFUs. Lung tissues were collected aseptically and analyzed for CFU counts, cytokine analysis, pulmonary cell analysis (flow cytometry analysis and lung cell restimulation), and histology. Spleens were collected aseptically for flow cytometry
Table 1 Primer sequences for qRT-PCR in mice

\begin{tabular}{|c|c|c|}
\hline Gene & & Sequence \\
\hline \multirow[t]{2}{*}{ GAPDH } & $\mathrm{F}$ & TGCCCAGAACATCATCCCTG \\
\hline & $\mathrm{R}$ & TCAGATCCACGACGGACACA \\
\hline \multirow[t]{2}{*}{ Def-B2 } & $\mathrm{F}$ & AAAGTATTGGATACGAAGCAGAACTTG \\
\hline & $\mathrm{R}$ & GGAGGACAAATGGCTCTGACA \\
\hline \multirow[t]{2}{*}{ Def-B3 } & $\mathrm{F}$ & TGAGGAAAGGAGGCAGATGCT \\
\hline & $\mathrm{R}$ & GGAACTCCACAACTGCCAATC \\
\hline \multirow[t]{2}{*}{ LL-37 } & $\mathrm{F}$ & CAGAGCGGCAGCTACCTGAG \\
\hline & $\mathrm{R}$ & TCACCACССССТGTТССТ \\
\hline \multirow[t]{2}{*}{ s100a9 } & $\mathrm{F}$ & CACCCTGAGCAAGAAGGAAT \\
\hline & $\mathrm{R}$ & TGTCATTATGAGGGCTTCATT \\
\hline \multirow[t]{2}{*}{$\operatorname{Reg}-3 \beta$} & $\mathrm{F}$ & ATGCTGCTCTCCTGCCTGATG \\
\hline & $\mathrm{R}$ & CTAATGCGTGCGGAGGGTATATTC \\
\hline \multirow[t]{2}{*}{ 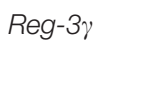 } & $\mathrm{F}$ & CTGTGGTACCCTGTCAAGAGC \\
\hline & $\mathrm{R}$ & GGCCTTGAATTTGCAGACAT \\
\hline \multirow[t]{2}{*}{$M M P-12$} & $\mathrm{~F}$ & GCAAAGGTGGTACAC \\
\hline & $\mathrm{R}$ & GCAACAAGGAAGAGG \\
\hline
\end{tabular}

analysis and restimulation culture analysis. Blood was used for the determination of CFU counts and cytokine analysis on serum.

Flow cytometry. Cells harvested from BALs and lungs were washed and incubated with antibodies (BD, Franklin lakes, NJ) for $30 \mathrm{~min}$ in phosphate-buffered saline before being washed. Staining was performed as described in Supplementary Information.

For intra-cellular staining, lung cells harvested were washed and incubated during $4 \mathrm{~h}$ with $3 \mu \mathrm{g} \mathrm{ml}^{-1}$ brefeldin A (eBioscience, San Diego, CA), and incubated with antibodies as described in Supplementary Information. Cells were analyzed on a LSR Fortessa (BD Biosciences). Gating strategies are reported in Supplementary Figure 1.

Cytokine and antimicrobial peptides measurement. Levels of IFN $-\gamma$, IL-1 $\beta$, IL-2, IL-6, IL-10, IL-13, IL-17, IL-22, and TNF- $\alpha$ were quantified in sera, lung tissue lysates, and BALs using commercial ELISA kits (eBiosciences; R\&D Systems, Minneapolis, MN). Similarly, levels of IFN- $\gamma$, IL-2, IL-13, IL-17, IL-22, and TNF- $\alpha$ were measured in the supernatants of lungs and spleen samples $\left(2 \times 10^{6}\right.$ of cells $)$ re-stimulated in vitro with HK NTHi during $72 \mathrm{~h}$.

To analyze the role of AhR in the default IL-22 production in COPD exacerbation by NTHi, levels of IFN- $\gamma$, IL-17, IL-22, and TNF- $\alpha$ were measured in the supernatants of spleen samples $\left(2 \times 10^{6}\right.$ of cells per $\mathrm{ml})$ re-stimulated in vitro with anti-CD3 antibody $\left(100 \mathrm{ng} \mathrm{ml}^{-1}\right.$, OKT3 clone), HK NTHi, and CSE (diluted $1 / 30$ or $1 / 60$ ) or $\mathrm{B}[\mathrm{a}] \mathrm{P}$ (Sigma-Aldrich, St Louis, MO; $250 \mathrm{ng} \mathrm{ml}^{-1}$ ) as a positive control during $72 \mathrm{~h}$.

Levels of S100A9, Def $\beta 2$, and REG3 $\gamma$ were quantified in lung tissue lysates and BALs using commercial ELISA kits (Boster, Pleasanton, CA; Abbexa, Cambridge, UK).

Primers. Quantitative RT-PCR was performed to quantify mRNA of interest (Table 1). Results were expressed as mean \pm s.e.m. of the relative gene expression calculated for each experiment in folds $\left(2^{-\Delta \Delta \mathrm{Ct}}\right)$ using GAPH as a reference, and compared with controls.

Histological analysis. To study lung remodeling post-infection with NTHi, the lungs were inflated and fixed in formalin. The lungs were paraffin-embedded; cross-sections were cut and stained with hematoxylin and eosin. Histopathologic score was defined as described in Supplementary Information. 
Statistical analysis. The data are expressed as mean \pm s.e.m. A MannWhitney unpaired $t$-test was used to compare two groups, unless otherwise specified. Comparisons of more than two groups were performed with a non-parametric Kruskal-Wallis one-way analysis of variance, followed by Dunn's post test (using PRISM software, v5 GraphPad). The threshold for statistical significance was set to $P<0.05$.

SUPPLEMENTARY MATERIAL is linked to the online version of the paper at http://www.nature.com/mi

\section{ACKNOWLEDGMENTS}

We gratefully acknowledge Eva Vilain and Gwenola Kervoaze for their excellent support in completion of experiments. We also acknowledge Dr JC Renauld (Brussels, Belgium), which has generated the IL-22deficient mice. We also thank Hélène Bauderlique for her help for cell sorting (BICel Cytométrie Plateform, Institut Pasteur de Lille, France). A special thanks to Jean-Claude Sirard and Isabelle Wolowczuk for their critical reviewing of the paper.

This work was granted by GlaxoSmithKline Biologicals SA. This work was supported by the Institut National de la Santé et de la Recherche Médicale (Inserm), the Centre National de la Recherche Scientifique (CNRS). Funders had no role in study design, data collection, data analysis, interpretation, and writing of the report.

\section{AUTHOR CONTRIBUTIONS}

Conception and design: R.S., M.P.C., M.P., and P.G.; analysis and interpretation: R.S., M.P.C., G.K., P.G., V.W., F.G., P.H., M.P., and P.G.; drafting the manuscript for important intellectual content: R.S., M.P.C., F.T., M.P., and P.G. All authors gave their agreement to be accountable for all aspects of the work in ensuring that questions related to the accuracy or integrity of any part of the work are appropriately investigated and resolved.

\section{DISCLOSURE}

V.W., F.G., and P.H. are employees of the GSK group of companies and own shares in GSK.

(c) 2017 Society for Mucosal Immunology

\section{REFERENCES}

1. Barnes, P.J. Immunology of asthma and chronic obstructive pulmonary disease. Nat. Rev. Immunol. 8, 183-192 (2008).

2. Pauwels, R.A. \& Rabe, K.F. Burden and clinical features of chronic obstructive pulmonary disease (COPD). Lancet Lond. Engl 364, 613-620 (2004).

3. Salvi, S.S. \& Barnes, P.J. Chronic obstructive pulmonary disease in nonsmokers. Lancet 374, 733-743 (2009).

4. Brusselle, G.G., Joos, G.F. \& Bracke, K.R. New insights into the immunology of chronic obstructive pulmonary disease. Lancet 378 , 1015-1026 (2011).

5. Pichavant, $M$. et al. Oxidative stress-mediated iNKT-cell activation is involved in COPD pathogenesis. Mucosal Immunol 7, 568-578 (2014).

6. Churg, A., Tai, H., Coulthard, T., Wang, R. \& Wright, J.L. Cigarette smoke drives small airway remodeling by induction of growth factors in the airway wall. Am. J. Respir. Crit. Care Med. 174, 1327-1334 (2006).

7. Hogg, J.C. et al. The nature of small-airway obstruction in chronic obstructive pulmonary disease. N. Engl. J. Med. 350, 2645-2653 (2004).

8. Ganesan, S. et al. Combined exposure to cigarette smoke and nontypeable Haemophilus influenzae drives development of a COPD phenotype in mice. Respir. Res. 15, 11 (2014).

9. Wedzicha, J.A. \& Donaldson, G.C. Exacerbations of chronic obstructive pulmonary disease. Respir. Care 48, 1204-1215 (2003).

10. Sethi, S., Evans, N., Grant, B.J.B. \& Murphy, T.F. New strains of bacteria and exacerbations of chronic obstructive pulmonary disease. N. Engl. J. Med. 347, 465-471 (2002).
11. Moghaddam, S.J., Ochoa, C.E., Sethi, S. \& Dickey, B.F. Nontypeable Haemophilus influenzae in chronic obstructive pulmonary disease and lung cancer. Int. J. Chron. Obstruct. Pulmon. Dis 6, 113-123 (2011).

12. Finney, L.J., Ritchie, A., Pollard, E., Johnston, S.L. \& Mallia, P. Lower airway colonization and inflammatory response in COPD: a focus on Haemophilus influenzae. Int. J. Chron. Obstruct. Pulmon. Dis 9, 1119-1132 (2014).

13. Murphy, T.F., Brauer, A.L., Schiffmacher, A.T. \& Sethi, S. Persistent colonization by Haemophilus influenzae in chronic obstructive pulmonary disease. Am. J. Respir. Crit. Care Med. 170, 266-272 (2004).

14. Cua, D.J. \& Tato, C.M. Innate IL-17-producing cells: the sentinels of the immune system. Nat. Rev. Immunol. 10, 479-489 (2010).

15. Barnes, P.J. Alveolar macrophages as orchestrators of COPD. COPD 1 , 59-70 (2004).

16. Pottelberge, G.R.V., Bracke, K.R., Joos, G.F. \& Brusselle, G.G. The role of dendritic cells in the pathogenesis of COPD: liaison officers in the front line. COPD 6, 284-290 (2009).

17. Freeman, C.M. et al. Lung CD8 + T cells in COPD have increased expression of bacterial TLRs. Respir. Res. 14, 13 (2013).

18. Essilfie, A.-T. et al. Haemophilus influenzae infection drives IL-17-mediated neutrophilic allergic airways disease. PLoS Pathog. 7, e1002244 (2011).

19. Kolls, J.K., McCray, P.B. \& Chan, Y.R. Cytokine-mediated regulation of antimicrobial proteins. Nat. Rev. Immunol. 8, 829-835 (2008).

20. Aujla, S.J. et al. IL-22 mediates mucosal host defense against Gramnegative bacterial pneumonia. Nat. Med 14, 275-281 (2008).

21. Gaschler, G.J. et al. Bacteria challenge in smoke-exposed mice exacerbates inflammation and skews the inflammatory profile. Am. J. Respir. Crit. Care Med. 179, 666-675 (2009).

22. Stockley, R.A. Neutrophils and the pathogenesis of COPD. Chest 121, 151S-155S (2002).

23. Barnes, P.J. The cytokine network in asthma and chronic obstructive pulmonary disease. J. Clin. Invest. 118, 3546-3556 (2008).

24. Lim, S. et al. Balance of matrix metalloprotease- 9 and tissue inhibitor of metalloprotease- 1 from alveolar macrophages in cigarette smokers. Am. J. Respir. Crit. Care Med. 162, 1355-1360 (2000).

25. Cheung, P.F., Wong, C.K. \& Lam, C.W. Molecular mechanisms of cytokine and chemokine release from eosinophils activated by IL-17A, IL-17F, and IL-23: implication for Th17 lymphocytes-mediated allergic inflammation. J. Immunol. 180, 5625-5635 (2008).

26. Ley, K., Smith, E. \& Stark, M.A. IL-17A-producing neutrophil-regulatory Tn Iymphocytes. Immunol. Res. 34, 229-242 (2006).

27. Roos, A.B. et al. IL-17A and the promotion of neutrophilia in acute exacerbation of chronic obstructive pulmonary disease. Am. J. Respir. Crit. Care Med. 192, 428-437 (2015).

28. Demedts, I.K. et al. Accumulation of dendritic cells and increased CCL2O levels in the airways of patients with chronic obstructive pulmonary disease. Am. J. Respir. Crit. Care Med. 175, 998-1005 (2007).

29. Barnes, P.J. Alveolar macrophages in chronic obstructive pulmonary disease (COPD). Cell. Mol. Biol. (Noisy-le-grand), 50 Online Pub, OL627-637 (2004).

30. Pichavant, M. et al. IL-22 defect during Streptococcus pneumoniae infection triggers exacerbation of chronic obstructive pulmonary disease. EBioMedicine 2, 1686-1696 (2015).

31. Plé, C. et al. Polycyclic aromatic hydrocarbons reciprocally regulate IL-22 and IL-17 cytokines in peripheral blood mononuclear cells from both healthy and asthmatic subjects. PLOS ONE 10, e0122372 (2015).

32. Eidenschenk, C., Rutz, S., Liesenfeld, O. \& Ouyang, W. Role of IL-22 in microbial host defense. Curr. Top. Microbiol. Immunol. 380, 213-236 (2014).

33. Rutz, S., Wang, X. \& Ouyang, W. The IL-20 subfamily of cytokines from host defence to tissue homeostasis. Nat. Rev. Immunol. 14, 783-795 (2014).

34. Ivanov, S. et al. Interleukin-22 reduces lung inflammation during influenza A virus infection and protects against secondary bacterial infection. J. Virol. 87, 6911-6924 (2013). 